\title{
CONTROLABILIDAD APROXIMADA SOBRE LA FRONTERA PARA LA ECUACIÓN SEMILINEAL DEL CALOR
}

\section{Víctor Rafael Cabanillas Zannini ${ }^{1}$}

RESUMEN.- Estudiamos el problema de la controlabilidad aproximada sobre la frontera asociado a la ecuación semilineal del calor

$$
\begin{cases}u^{\prime}-\Delta u+f(u)=0 & , \text { en } Q \\ u=v & , \text { sobre } \Sigma \\ u(0)=u^{0} & , \text { en } \Omega\end{cases}
$$

Demostramos que, dados $u^{0}, u^{1} \in H^{-1}(\Omega)$ es posible construir un control $v \in L^{2}\left(0, T ; L^{2}(\Gamma)\right)$ que conduce a la solución u arbitrariamente cerca del estado objetivo $u^{1}$.

El trabajo presenta una adaptación a problemas de control frontera de una técnica de punto fijo introducida por E. Zuazua en el contexto de la ecuación de la onda, y que había sido aplicada también en el estudio de la controlabilidad aproximada cuando los controles actúan en el interior del dominio $\Omega$.

La demostración que presentamos es contructiva en el sentido de que obtenemos explícitamente un control frontera en función de la solución del sistema adjunto asociado a nuestro problema.

PALABRAS CLAVE.- Controlabilidad aproximada. Control frontera. Continuación única. Sistema adjunto.

\section{APPROXIMATE BOUNDARY CONTROLLABILITY FOR THE SEMILINEAR HEAT EQUATION}

ABSTRACT.- We study the problem of the approximate boundary controllability associated to the semilinear heat equation

$$
\begin{cases}u^{\prime}-\Delta u+f(u)=0 & , \text { in } Q \\ u=v & , \text { on } \Sigma \\ u(0)=u^{0} & , \text { in } \Omega\end{cases}
$$

We show that, given $u^{0}, u^{1} \in H^{-1}(\Omega)$ it is possible to build a control $v \in L^{2}\left(0, T ; L^{2}(\Gamma)\right)$ that guide to the solution or arbitrarily near the objective state $u^{1}$.

\footnotetext{
' Universidad Nacional Mayor de San Marcos. Facultad de Ciencias Matemáticas. e-mail: vcabanillasz@unmsm.edu.pe
} 
The work presents an adaptation for boundary control problems of a fixed point technique introduced by E. Zuazua in the context of the equation of the wave, which had also been applied in the study of the approximate controlability when the controls act inside the domain $\Omega$.

The demonstration that we present is constructive in the sense that we explicitly obtain a boundary control in function of the solution of the adjoint system associated to our problem.

KEY WORDS.- Approximate controllability. Boundary control. Unique continuation. Adjoint system.

\section{INTRODUCCIÓN}

Sea $\Omega$ un dominio acotado de $\mathbb{R}^{n}, n \geq 1$, con frontera $\Gamma$ de clase $C^{2}$. Denotemos por $Q$ al cilindro $\Omega \times(0, T)$ y por $\Sigma$ su frontera lateral, es decir $Q=\Omega \times(0, T)$ y $\Sigma=\Gamma \times(0, T)$. Consideremos el problema de valor inicial, con condición de frontera no homogénea para la ecuación semilineal del calor

$$
\left\{\begin{array}{ll}
u^{\prime}-\Delta u+f(u)=0 & , \text { en } Q \\
u=v & , \text { sobre } \Sigma \\
u(0)=u^{0} & , \text { en } \Omega
\end{array} .\right.
$$

Supongamos que la no-linealidad $f: \mathbb{R} \rightarrow \mathbb{R}$ es tal que

$f \in W^{1, \infty}(\mathbb{R})$, y es globalmente Lipschitz, i.e., existe $L>0$ tal que

$$
|f(y)-f(z)| \leq L|y-z| \forall y, z \in \mathbb{R}
$$

En el presente trabajo estudiamos el problema de la controlabilidad en la frontera para el sistema (1.1), propuesto por E. Zuazua en [9]. Probaremos que el sistema (1.1) es aproximadamente controlable cuando el control actúa sobre la frotera $\Gamma$. El caso en el que el control actúa sobre el interior de $\Omega$, es decir, la controlabilidad interna para el sistema (1.1), fue estudiado por L.A. Fernández y E. Zuazua en [4] por medio de argumentos de problemas de control optimal y posteriormente por E. Zuazua en [9] por medio de una adaptación del método de punto fijo que él mismo introdujo en el contexto de la ecuación de la onda en [7].

Siguiendo las ideas expuestas en [9] demostramos la controlabilidad aproximada en $H^{-1}(\Omega)$ del sistema (1.1).

\section{Formulación del problema de control}

El problema de la controlabilidad aproximada sobre la frontera asociado a (1.1) puede ser formulado de la siguiente manera:

Dados $T>0, u^{0}, u^{1} \in H^{-1}(\Omega)$ y $\varepsilon>0$, hallar un control $v \in L^{2}\left(0, T ; L^{2}(\Gamma)\right)$ tal que la solución u de (1.1) satisfaga

$$
\left\|u(T)-u^{1}\right\|_{H^{-1}(\Omega)}<\varepsilon
$$


La función $u$ representa el estado del sistema, $u^{0}$ es la condición inicial del problema, $u^{1}$ es la función objetivo, es decir, estado que se quiere alcanzar después de un tiempo $T$. Es claro que dada una condición inicial $u^{0}$ y fijando un estado $u^{1}$, nada garantiza que después de un tiempo $T$ el estado $u(T)=u(x, T)$ del sistema se encuentre próximo de $u^{1}$, por tal razón, para lograr que, pasado un tiempo $T$ el estado se encuentre a una distancia de $u^{1}$ menor que $\varepsilon$, es necesario actuar sobre el sistema a través de un control, al que hemos denotado por $v$. Cuando este control $v$ actúa sobre el interior de $\Omega$ se dice que $v$ es un control interno, en cambio, si $v$ actúa sobre la frontera $\Gamma \operatorname{de} \Omega$ decimos que $v$ es un control frontera. Nuestro problema es de control frontera y consiste en encontrar una función $v$ que actúe sobre la frontera de $\Omega$ y que conduzca a la solución $u$ del sistema arbitrariamente cerca del objetivo $u^{1}$.

El objetivo de la presente nota es mostrar como la estrategia de punto fijo, utilizada por E. Zuazua en [9] para el estudio de la controlabilidad interna, puede ser adaptada para el problema de la controlabilidad en la frontera.

El siguiente resultado garantiza la existencia y unicidad de las soluciones del sistema (1.1).

Proposición 1.1 Suponga que $f$ satisface (1.2). Entonces para cualquier $u^{0} \in H^{-1}(\Omega)$ y $v \in H_{0}^{1}\left(0, T ; H^{-1 / 2}(\Gamma)\right) \cap L^{2}\left(0, T ; H^{1 / 2}(\Gamma)\right)$ existe una única solución del sistema (1.1) en la clase

$$
v \in C\left([0, T] ; H^{-1}(\Omega)\right) \cap L^{2}\left(0, T ; L^{2}(\Omega)\right)
$$

Demostración. Ver J.L. Lions - E. Magenes [6].

Observación 1.1. Si $u^{0} \in L^{2}(\Omega)$ en la Proposición 1.1 entonces la solución $u$ de (1.1) pertenece a la clase $u \in C\left([0, T] ; L^{2}(\Omega)\right) \cap L^{2}\left(0, T ; H^{1}(\Omega)\right)$.

\section{DESCRIPCIÓN DEL MÉTODO DE PUNTO FIJO}

Como mencionamos en la sección anterior, el método que usaremos para estudiar la controlabilidad del sistema (1.1) es una adaptación del método de punto fijo introducido por E. Zuazua [7] en el contexto de la ecuación de la onda.

Observamos que, para cualquier $y \in L^{2}\left(0, T ; H^{-1}(\Omega)\right)$ la siguiente identidad es consecuencia del Teorema Fundamental del Cálculo

$$
f(y)-f(0)=\int_{0}^{1} \frac{d}{d \sigma} f(\sigma y) d \sigma=\int_{0}^{1} \frac{\partial}{\partial y} f(\sigma y) d \sigma y .
$$

Denotemos

$$
F(y)=\int_{0}^{1} \frac{\partial}{\partial y} f(\sigma y) d \sigma
$$


En vista de la hipótesis sobre $f, F$ aplica $L^{2}\left(0, T ; H^{-1}(\Omega)\right)$ en un conjunto acotado de $L^{\infty}(Q)$. Más aún,

siendo $L$ la constante de Lipschitz de $f$.

$$
\|F(y)\|_{L^{\infty}(Q)} \leq L
$$

Usando estas notaciones, el sistema (1.1) puede reescribirse de la siguiente manera

$$
\begin{cases}u^{\prime}-\Delta u+F(u) u+f(0)=0 & , \text { en } Q \\ u=v & , \text { sobre } \Sigma \\ u(0)=u^{0} & , \text { en } \Omega\end{cases}
$$

Dado $y \in L^{2}\left(0, T ; H^{-1}(\Omega)\right)$ consideremos ahora el sistema linealizado

$$
\begin{cases}u^{\prime}-\Delta u+F(y) u+f(0)=0 & , \text { en } Q \\ u=v & , \text { sobre } \Sigma \\ u(0)=u^{0} & , \text { en } \Omega\end{cases}
$$

Observemos que (2.5) es un sistema lineal en relación al estado $u$ cuyo potencial $a=F(y)$ satisface la condición de acotación uniforme

$$
\|a\|_{L^{\infty}(Q)} \leq L
$$

Con esta notación, el sistema (2.5) puede reescribirse como sigue

$$
\begin{cases}u^{\prime}-\Delta u+a u+f(0)=0 & , \text { en } Q \\ u=v & , \text { sobre } \Sigma \\ u(0)=u^{0} & , \text { en } \Omega\end{cases}
$$

Ahora, fijemos el dato inicial $u^{0} \in H^{-1}(\Omega)$, el objetivo $u^{1} \in H^{-1}(\Omega)$ y un número real $\varepsilon>0$.

J.L. Lions introdujo en [5] un enfoque variacional para el problema de la controlabilidad aproximada, el mismo que también fue desarrollado por C. Fabre, J. P. Puel y E. Zuazua en [2] y adaptado al problema de la controlabilidad finito-aproximada por E. Zuazua en [8]. Adaptando este enfoque variacional para el caso de contrabilidad en la frontera, construiremos un control $v \in L^{2}\left(0, T ; L^{2}(\Gamma)\right)$ para el sistema lineal (2.7) tal que la solución $u$ de este sistema satisfaga

$$
\left\|u(T)-u^{1}\right\|_{H^{-1}(\Omega)}<\varepsilon
$$

El control $v \in L^{2}\left(0, T ; L^{2}(\Gamma)\right)$ satisfaciendo (2.8) no es único, sin embargo los argumentos variacionales mencionados antes premiten construir un control $v$ que tiene unicidad en el sentido de que es el único control con norma $L^{2}\left(0, T ; L^{2}(\Gamma)\right)$ mínima. 
De esta forma, para cualquier $y \in L^{2}\left(0, T ; H^{-1}(\Omega)\right)$ construimos un control $v=v(x, t ; y) \in L^{2}\left(0, T ; L^{2}(\Gamma)\right)$ tal que la solución $u=u(x, t ; y) \in C\left([0, T] ; H^{-1}(\Omega)\right) \cap L^{2}\left(0, T ; L^{2}(\Omega)\right)$ de (2.7) satisface (2.8). Esto permite definir una aplicación no lineal

$$
\begin{gathered}
\mathcal{N}: L^{2}\left(0, T ; H^{-1}(\Omega)\right) \rightarrow L^{2}\left(0, T ; H^{-1}(\Omega)\right) \\
\mathcal{N}(y)=u(x, t ; y)
\end{gathered}
$$

Vemos entonces que, el problema de la controlabilidad del sistema (1.1) se reduce a encontrar un punto fijo para la aplicación $\mathcal{N}$. En efecto, si $y \in L^{2}\left(0, T ; H^{-1}(\Omega)\right)$ es un punto fijo $\mathcal{N}$, i.e. $\mathcal{N}(y)=u=y$, la solución $u$ de (2.7) es en realidad solución de (2.4), pues $a=F(y)=F(u)$ y $F(u) u+f(0)=f(u)$. Entonces el control $v=v(y)$ que encontramos para el sistema linealizado (2.7) es el que también permite controlar el sistema no lineal (1.1) y hace que la solución de (1.1) satisfaga (2.8).

Como veremos más adelante, la aplicación no lineal $\mathcal{N}$ satisface las siguientes tres propiedades

i. $\mathcal{N}: L^{2}\left(0, T ; H^{-1}(\Omega)\right) \rightarrow L^{2}\left(0, T ; H^{-1}(\Omega)\right)$ es continua,

ii. $\mathcal{N}: L^{2}\left(0, T ; H^{-1}(\Omega)\right) \rightarrow L^{2}\left(0, T ; H^{-1}(\Omega)\right)$ es compacta,

iii. Existe una constante $R>0$ tal que

$$
\|\mathcal{N}(y)\|_{L^{2}\left(0, T ; H^{-1}(\Omega)\right)} \leq R, \forall y \in L^{2}\left(0, T ; H^{-1}(\Omega)\right)
$$

En vista de estas propiedades, la existencia de un punto fijo para $\mathcal{N}$ es una consecuencia inmediata del Teorema de Punto Fijo de Schauder.

\section{CONTROLABILIDAD DEL SISTEMA LINEALIZADO}

Dado el potencial $a \in W^{1, \infty}(Q)$ y una constante real $\lambda$, consideremos el problema de control frontera

$$
\begin{cases}u^{\prime}-\Delta u+a u+\lambda=0 & , \text { en } Q \\ u=v & , \text { sobre } \Sigma \\ u(0)=u^{0} & , \text { en } \Omega\end{cases}
$$

Dados $u^{0}, u^{1} \in H^{-1}(\Omega)$ y $\varepsilon>0$, buscamos un control $v \in L^{2}\left(0, T ; L^{2}(\Gamma)\right)$ tal que la solución $u$ de (3.1) satisfaga

$$
\left\|u(T)-u^{1}\right\|_{H^{-1}(\Omega)}<\varepsilon
$$


En relación a este problema tenemos el siguiente teorema, que constituye el resultado central del trabajo.

Teorema 3.1 Sean $T>0, y u^{0}, u^{1} \in H^{-1}(\Omega)$. Entonces, existe un control $v \in L^{2}\left(0, T ; L^{2}(\Gamma)\right)$ tal que la solución $u \in C\left([0, T] ; H^{-1}(\Omega)\right) \cap L^{2}\left(0, T ; L^{2}(\Omega)\right)$ de (3.1) satisface (3.2). Además, para cualquier $R>0$ existe una constante $C(R)>0$ tal que

$$
\|v\|_{L^{2}\left(0, T ; L^{2}(\Gamma)\right)} \leq C(R)
$$

para cualquier potencial $a \in W^{1, \infty}(Q)$ satisfaciendo

$$
\|a\|_{W^{1, \infty}(Q)} \leq R
$$

\section{Demostración del Teorema 3.1}

Sin pérdida de generalidad podemos asumir que $\lambda=0 \quad$ y $\quad u^{0}=0$. En consecuencia, en adelante estudiaremos la controlabilidad en la frontera del sistema

$$
\begin{cases}u^{\prime}-\Delta u+a u=0 & , \text { en } Q \\ u=v & , \text { sobre } \Sigma \\ u(0)=0 & , \text { en } \Omega\end{cases}
$$

Consideremos el sistema adjunto

$$
\begin{cases}-\varphi^{\prime}-\Delta \varphi+a \varphi=0 & , \text { en } Q \\ \varphi=0 & , \text { sobre } \Sigma \\ \varphi(T)=\varphi^{0} & , \text { en } \Omega\end{cases}
$$

Notemos que (3.6) es un problema parabólico definido sobre $(0, T)$ cuyo dato inicial es $\varphi(T)=\varphi^{0}$, es decir, $t=T$ es el tiempo inicial y $t=0$ el tiempo final, por esta razón, se dice que (3.6) es un problema retrógrado en el tiempo.

Teniendo en cuenta que el potencial $a$ es acotado, si $\varphi^{0} \in H_{0}^{1}(\Omega)$, el sistema (3.6) tiene una única solución en la clase

$$
\varphi \in C\left([0, T], H_{0}^{1}(\Omega)\right) \cap L^{2}\left(0, T ; H^{2}(\Omega) H_{0}^{1}(\Omega)\right)
$$

Observemos que al ser $\varphi$ una función de $L^{2}\left(0, T ; H^{2}(\Omega) \cap H_{0}^{1}(\Omega)\right)$, la derivada normal sobre $\Gamma$ tiene sentido y gracias al Teorema de la Traza resulta que 


$$
\frac{\partial \varphi}{\partial \nu} \in L^{2}\left(0, T ; L^{2}(\Gamma)\right)
$$

Ahora, consideremos el funcional $J: H_{0}^{1}(\Omega) \rightarrow \mathbb{R}$ definido por

$$
J\left(\varphi^{0}\right)=\frac{1}{2} \int_{0}^{T} \int_{\Gamma}\left(\frac{\partial \varphi}{\partial \nu}\right)^{2} d \Gamma d t+\varepsilon\left\|\varphi^{0}\right\|_{H_{0}^{1}(\Omega)}-\left\langle u^{1}, \varphi^{0}\right\rangle_{H^{-1}(\Omega) \times H_{0}^{1}(\Omega)}
$$

donde $\varphi$ denota la solución del sistema (3.6) con dato inicial $\varphi^{0}$.

No es difícil demostrar que

$$
\begin{aligned}
& J: H_{0}^{1}(\Omega) \rightarrow \mathbb{R} \text { es continuo } \\
& J: H_{0}^{1}(\Omega) \rightarrow \mathbb{R} \text { es convexo }
\end{aligned}
$$

Más aún,

$$
J: H_{0}^{1}(\Omega) \rightarrow \mathbb{R} \text { es estrictamente convexo }
$$

Esta última propiedad es consecuencia de un resultado de continuación única debido a C. Fabre [3]. La continuación única también permite demostrar que

$$
J: H_{0}^{1}(\Omega) \rightarrow \mathbb{R} \text { es coercivo }
$$

Probaremos las coercividad de $J$ en la siguiente proposición.

Las propiedades anteriores del funcional $J$ permiten concluir que el problema variacional

$$
\min _{\varphi^{0} \in H_{0}^{1}(\Omega)} J\left(\varphi^{0}\right)
$$

posee una única solución, i.e., el funcional $J$ alcanza su mínimo en un único elemento de $H_{0}^{1}(\Omega)$.

La propiedad de coercividad anunciada se demuestra en la siguiente:

Proposición 3.2 En las condiciones y notaciones anteriores se tiene

$$
\liminf _{\left\|\varphi^{0}\right\|_{H_{0}^{1}(\Omega)} \rightarrow \infty} \frac{J\left(\varphi^{0}\right)}{\left\|\varphi^{0}\right\|_{H_{0}^{1}(\Omega)}} \geq \varepsilon
$$

Demostración. La demostración de esta proposición sigue las ideas y técnicas de [2] combinadas con el resultado de continuación única de C. Fabre [3]. Sea $\left(\varphi_{n}^{0}\right)_{n}$ una sucesión en $H_{0}^{1}(\Omega)$ tal que $\left\|\varphi^{0}\right\|_{H_{0}^{1}(\Omega)} \rightarrow \infty$ y denotemos

$$
\hat{\varphi}_{n}^{0}=\frac{\varphi_{n}^{0}}{\left\|\varphi_{n}^{0}\right\|_{H_{0}^{1}(\Omega)}} \quad, \quad \hat{\varphi}_{n}=\frac{\varphi_{n}}{\left\|\varphi_{n}^{0}\right\|_{H_{0}^{1}(\Omega)}}
$$


siendo $\varphi_{n}$ la solución de (3.6) con dato inicial $\varphi_{n}^{0}$, entonces $\widehat{\varphi_{n}}$ es solución de (3.6) con dato normalizado $\hat{\varphi}_{n}^{0}$.

En lo que sigue, $|$.$| y \|\cdot\|$ denotarán las normas en $L^{2}(\Omega)$ y $H_{0}^{1}(\Omega)$ respectivamente, y $\langle$,$\rangle la dualidad H^{-1}(\Omega) \times H_{0}^{1}(\Omega)$.

De la definición de $J$ tenemos

$$
\frac{J\left(\varphi_{n}^{0}\right)}{\left\|\varphi_{n}^{0}\right\|}=\frac{\left\|\varphi_{n}^{0}\right\|}{2} \int_{0}^{T} \int_{\Gamma}\left(\frac{\partial \hat{\varphi}_{n}}{\partial v}\right)^{2} d \Gamma d t+\varepsilon\left\|\hat{\varphi}_{n}^{0}\right\|-\left\langle u^{1}, \hat{\varphi}_{n}^{0}\right\rangle
$$

Como queremos estimar $\lim _{\left\|\varphi^{0}\right\|_{H_{0}^{1}(\Omega)} \rightarrow \infty} \frac{J\left(\varphi^{0}\right)}{\left\|\varphi^{0}\right\|_{H_{0}^{1}(\Omega)}}$, al tomar límite inferior en la identidad anterior, se presentan dos casos:

Caso 1.

$$
\liminf _{n \rightarrow \infty} \int_{0}^{T} \int_{\Gamma}\left(\frac{\partial \hat{\varphi}_{n}}{\partial v}\right)^{2} d \Gamma d t>0
$$

Caso 2.

$$
\liminf _{n \rightarrow \infty} \int_{0}^{T} \int_{\Gamma}\left(\frac{\partial \hat{\varphi}_{n}}{\partial \nu}\right)^{2} d \Gamma d t=0
$$

En el primer caso, el término

$$
\frac{\left\|\varphi_{n}^{0}\right\|}{2} \int_{0}^{T} \int_{\Gamma}\left(\frac{\partial \hat{\varphi}_{n}}{\partial \nu}\right)^{2} d \Gamma d t
$$

de (3.12) tiende $a+\infty$ mientras que los dos términos restantes permanecen acotados, en consecuencia (3.10) es cierto.

Analicemos el segundo caso. Consideremos una subsucesión, a la cual, con el fin de simplificar la notación, denotaremos con el mismo subíndice $n$, tal que

$$
\lim _{n \rightarrow \infty} \int_{0}^{T} \int_{\Gamma}\left(\frac{\partial \hat{\varphi}_{n}}{\partial v}\right)^{2} d \Gamma d t=0
$$

entonces, para alguna subsucesión, podemos concluir que

$$
\hat{\varphi}_{n}^{0} \rightarrow \hat{\varphi}^{0} \text { débilmente en } H_{0}^{1}(\Omega)
$$


Sean $\hat{\varphi}_{n}$ y $\hat{\varphi}$ soluciones de (3.6) correspondientes a los datos iniciales $\hat{\varphi}_{n}^{0}$ y $\hat{\varphi}^{0}$, entonces $\hat{\varphi}_{n}$ y $\hat{\varphi}$ tienen la regularidad (3.7).

Sea $z \in C\left([0, T] ; L^{2}(\Omega)\right) \cap L^{2}\left(0, T ; H^{1}(\Omega)\right)$ solución de

$$
\begin{cases}z^{\prime}-\Delta z+a z=0 & , \text { en } Q \\ z=w & , \text { sobre } \Sigma \\ z(0)=0 & , \text { en } \Omega\end{cases}
$$

con $w \in \mathscr{C}=H_{0}^{1}\left(0, T ; H^{-1 / 2}(\Gamma)\right) \cap L^{2}\left(0, T ; H^{1 / 2}(\Gamma)\right)$. Multiplicando la ecuación (3.6) por $z$ e integrando obtenemos

$$
\left\langle\frac{\partial \varphi}{\partial v}, w\right\rangle_{L^{2}\left(0, T ; H^{1 / 2}(\Gamma)\right) \times L^{2}\left(0, T ; H^{-1 / 2}(\Gamma)\right)}=-(\varphi(T), z(T))
$$

Entonces, para $\hat{\varphi}_{n}$ y $\hat{\varphi}$ soluciones de (3.6) con datos iniciales $\hat{\varphi}_{n}^{0}$ y $\hat{\varphi}^{0}$ respectivamente, tenemos

$$
\left\langle\frac{\partial \hat{\varphi}_{n}}{\partial v}-\frac{\partial \hat{\varphi}}{\partial v}, w\right\rangle=-\left(\hat{\varphi}_{n}^{0}-\hat{\varphi}^{0}, z(T)\right)
$$

Por (3.16) y la densidad de $\mathscr{H}$ en $L^{2}\left(0, T ; H^{-1 / 2}(\Gamma)\right)$ tenemos

$$
\frac{\partial \hat{\varphi}_{n}}{\partial \nu} \rightarrow \frac{\partial \hat{\varphi}}{\partial \nu} \text { débilmente en } L^{2}\left(0, T ; H^{1 / 2}(\Gamma)\right)
$$

en consecuencia, por (3.15) concluimos que

$$
\frac{\partial \hat{\varphi}}{\partial v}=0 \text { en casi todo punto de } \Sigma
$$

entonces, por la propiedad de continuación única de C. Fabre [3] concluimos que $\hat{\varphi} \equiv 0$ y consecuentemente $\hat{\varphi}^{0}=0$.

Entonces por (3.16)

$$
\hat{\varphi}_{n}^{0}>0 \text { débilmente en } H_{0}^{1}(\Omega)
$$

luego, como $u^{1} \in H^{-1}(\Omega)$, tomando $\lim \inf$ en (3.12), y ya que $\left\|\hat{\varphi}_{n}^{0}\right\|=1$ tenemos

$$
\liminf _{n \rightarrow \infty} \frac{J\left(\varphi_{n}^{0}\right)}{\left\|\varphi_{n}^{0}\right\|} \geq \varepsilon
$$

lo cual completa la demostración de la proposición. 
Retornemos a la demostración del Teorema 3.1.

Siendo $J: H_{0}^{1}(\Omega) \rightarrow \mathbb{R}$ continuo, estrictamente convexo y coercivo, entonces $J$ alcanza su mínimo en un único elemento $\hat{\varphi}^{0} \in H_{0}^{1}(\Omega)$, i.e. $\hat{\varphi}^{0}$ satisface

$$
J\left(\hat{\varphi}^{0}\right)=\min _{\varphi^{0} \in H_{0}^{1}(\Omega)} J\left(\varphi^{0}\right)
$$

Entonces, consideremos el control

$$
v=-\frac{\partial \hat{\varphi}}{\partial v}
$$

siendo $\hat{\varphi}$ la solución del sistema adjunto (3.6) teniendo al minimizante $\hat{\varphi}^{0}$ como dato inicial. Entonces, $\hat{\varphi}$ tiene la regularidad (3.7), en consecuencia, por el Teorema de la Traza, $\frac{\partial \hat{\varphi}}{\partial \nu} \in L^{2}\left(0, T ; H^{1 / 2}(\Gamma)\right)$.

Ahora veamos que el control $v$ definido en (3.23) es el que permite obtener la controlabilidad aproximada que se buscaba, i.e., debe ser tal que la solución $u \in C\left([0, T] ; L^{2}(\Omega)\right) \cap L^{2}\left(0, T ; H^{1}(\Omega)\right)$ de

$$
\begin{cases}u^{\prime}-\Delta u+a u=0 & , \text { en } Q \\ u=-\frac{\partial \hat{\varphi}}{\partial \nu} & , \text { sobre } \Sigma \\ u(0)=0 & , \text { en } \Omega\end{cases}
$$

debe satisfacer

$$
\left\|u(T)-u^{1}\right\|_{H^{-1}}<\varepsilon
$$

En efecto, dado cualquier $\psi^{0} \in H_{0}^{1}(\Omega)$ y $\lambda \in \mathbb{R}$ tenemos

$$
J\left(\hat{\varphi}^{0}\right) \leq J\left(\hat{\varphi}^{0}-\lambda \psi^{0}\right)
$$

Dividiendo esta desigualdad por $\lambda>0$ y haciendo $\lambda \rightarrow 0^{+}$obtenemos que

$$
-\left\langle u^{1}, \psi^{0}\right\rangle+\int_{0}^{T} \int_{\Gamma} \frac{\partial \hat{\varphi}}{\partial v} \frac{\partial \psi}{\partial v} d \Gamma d t \leq \varepsilon\left\|\psi^{0}\right\|
$$

Reproduciendo este argumento con $\lambda<0$ obtenemos finalmente que

$$
\left|\left\langle u^{1}, \psi^{0}\right\rangle-\int_{0}^{T} \int_{\Gamma} \frac{\partial \hat{\varphi}}{\partial v} \frac{\partial \psi}{\partial \nu} d \Gamma d t\right| \leq \varepsilon\left\|\psi^{0}\right\|
$$

donde $\psi$ es solución de (3.6) con dato inicial $\psi^{0}$. 
Por otra parte, multiplicando (3.24) por $\psi$ e integrando por partes deducimos que

$$
\left\langle u(T), \psi^{0}\right\rangle=\int_{0}^{T} \int_{\Gamma} \frac{\partial \hat{\varphi}}{\partial v} \frac{\partial \psi}{\partial v} d \Gamma d t
$$

Combinando (3.28) y (3.27) llegamos a

$$
\left|\left\langle u(T)-u^{1}, \psi^{0}\right\rangle\right| \leq \varepsilon\left\|\psi^{0}\right\|, \forall \psi^{0} \in H_{0}^{1}(\Omega)
$$

lo cual es equivalente a (3.25).

Con el fin de probar la acotación uniforme (3.3) de los controles, primero observamos que el problema puede ser reducido al caso $u^{0}=0$ y $\lambda=0$, desde que $u^{1}$ se encuentre variando en un conjunto relativamente compacto de $H^{-1}(\Omega)$.

Al respecto, tenemos la siguiente proposición.

Proposición 3.3 Sean $R>0$ y $K$ un conjunto relativamente compacto de $H^{-1}(\Omega)$. Entonces, la propiedad de coercividad (3.i0) vale uniformemente sobre $u^{1} \in K$ y un potencial a satisfaciendo la limitación (3.4).

Observación 3.1 Que la propiedad de coercividad (3.10) valga uniformemente sobre $u^{1} \in K$ y el potencial $a \in W^{1, \infty}(Q)$ significa que para cada $j \in \mathbb{N}$, si $u_{j}^{1} \in K$ y si $a_{j}$ es un potencial en $W^{1, \infty}(Q)$ satisfaciendo

$$
\left\|a_{j}\right\|_{W^{1, \infty}(Q)} \leq R
$$

entonces, dado $\varepsilon>0$, se tiene

$$
\lim _{\left\|\varphi_{j}^{0}\right\|_{H_{0}^{1}(\Omega)} \rightarrow \infty} \frac{J_{j}\left(\varphi_{j}^{0}\right)}{\left\|\varphi_{j}^{0}\right\|_{H_{0}^{1}(\Omega)}} \geq \varepsilon
$$

donde $J_{j}$ es el funcional asociado a $u_{j}^{1}$ y $a_{j}$.

Observación 3.2. Como una consecuencia de la Proposición 3.3 deducimos que los minimizantes de los funcionales $J_{j}$ son uniformemente acotados cuando $u^{1} \in K$ y el potencial a es uniformemente acotado. Consecuentemente, los controles $v=-\frac{\partial \hat{\varphi}}{\partial v}$ son uniformemente acotados.

La demostración de la Proposición 3.3 es similar a la dada para la Proposición 3.2. Esto completa la demostración del Teorema 3.1. 
Observación 3.3. La regularidad del control $v$ puede ser mejorada. En efecto, es posible demostrar, usando argumentos de regularidad para problemas elípticos que $v=-\frac{\partial \hat{\varphi}}{\partial v} \in H^{1}\left(0, T ; H^{1 / 2}(\Gamma)\right)$.

\section{CONTROL DEL PROBLEMA SEMILINEAL}

Teorema 4.1 Supongamos que $f$ satisface (1.2). Entonces para todo $T>0$, el sistema (1.1) es aproximadamante controlable en $H^{-1}(\Omega)$. Más precisamente, para cualquier $u^{0}, u^{1} \in H^{-1}(\Omega)$ y $\varepsilon>0$ existe un control $v \in L^{2}\left(0, T ; L^{2}(\Gamma)\right)$ tal que la solución u de (1.1) satisface (1.3).

Observación 4.1. En realidad, el control $v$ del Teorema 4.1 pertenece a $H^{1}\left(0, T ; H^{1 / 2}(\Gamma)\right)$, desde que el control del Teorema 4.1 es el mismo que el obtenido en el Teorema 3.1 para el sistema linealizado. Ver observación 3.3.

\section{Demostración del Teorema 4.1.}

Dados $u^{0}, u^{1} \in H^{-1}(\Omega)$ y $\varepsilon>0$, la aplicación no lineal

$$
\begin{gathered}
\mathcal{N}: L^{2}\left(0, T ; H^{-1}(\Omega)\right) \rightarrow L^{2}\left(0, T ; H^{-1}(\Omega)\right) \\
\mathcal{N}(y)=u(x, t ; y)
\end{gathered}
$$

que a cada $y \in L^{2}\left(0, T ; H^{-1}(\Omega)\right)$ asocia la función $\mathcal{N}(y)=u(x, t ; y)$ solución de $(2.7)$ con potencial $a=F(y)$ definido en (2.2), está bien definida en vista del Terorema 3.1.

Como indicamos en la sección 3, con el fin de concluir la existencia de un punto fijo para $\mathcal{N}$ por medio del Teorema de Punto Fijo de Schauder es suficiente probar que $\mathcal{N}$ satisface las siguientes tres propiedades:

i. $\mathcal{N}: L^{2}\left(0, T ; H^{-1}(\Omega)\right) \rightarrow L^{2}\left(0, T ; H^{-1}(\Omega)\right)$ es continua,

ii. $\mathcal{N}: L^{2}\left(0, T ; H^{-1}(\Omega)\right) \rightarrow L^{2}\left(0, T ; H^{-1}(\Omega)\right)$ es compacta,

iii. Existe una constante $R>0$ tal que

$$
\|\mathcal{N}(y)\|_{L^{2}\left(0, T ; H^{-1}(\Omega)\right)} \leq R, \forall y \in L^{2}\left(0, T ; H^{-1}(\Omega)\right)
$$

\section{Continuidad de $\mathcal{N}$ :}

Sea $\left(y_{j}\right)_{j}$ una sucesión en $L^{2}\left(0, T ; H^{-1}(\Omega)\right)$ tal que $y_{j} \rightarrow y$ en $L^{2}\left(0, T ; H^{-1}(\Omega)\right)$.

Denotemos $\mathcal{N}\left(y_{j}\right)=u_{j}$, donde $u_{j}$ es solución de

$$
\begin{cases}u_{j}^{\prime}-\Delta u_{j}+F\left(y_{j}\right) u_{j}+f(0)=0 & , \text { en } Q \\ u_{j}=v_{j} & , \text { sobre } \Sigma \\ u_{j}(0)=u^{0} & , \text { en } \Omega\end{cases}
$$


Para concluir la continuidad de $\mathcal{N}$ es suficiente comprobar que el límite $\hat{\varphi}^{0}$ en (4.3) es el minimizante del funcional $J$ asociado al problema de control límite (4.6) - (4.7). Para hacer esto, dado $\psi^{0} \in H_{0}^{1}(\Omega)$, tenemos que mostrar que

$$
J\left(\hat{\varphi}^{0}\right) \leq J\left(\psi^{0}\right)
$$

Pero esto es inmediato, desde que, por la semicontinuidad inferior de $J$, tenemos

$$
J\left(\hat{\varphi}^{0}\right) \leq \liminf _{j \rightarrow \infty} J_{j}\left(\hat{\varphi}_{j}^{0}\right)
$$

por otra parte

$$
J\left(\psi^{0}\right) \leq \lim _{j \rightarrow \infty} J_{j}\left(\psi^{0}\right)
$$

además, como $\hat{\varphi}_{j}^{0}$ es el elemento minimizante de $J_{j}$ resulta que

$$
J_{j}\left(\hat{\varphi}_{j}^{0}\right) \leq J_{j}\left(\psi^{0}\right)
$$

Luego

$$
J\left(\hat{\varphi}^{0}\right) \leq \liminf _{j \rightarrow \infty} J_{j}\left(\hat{\varphi}_{j}^{0}\right) \leq \liminf _{j \rightarrow \infty} J_{j}\left(\psi^{0}\right)=J\left(\psi^{0}\right)
$$

como se quería.

\section{Compacidad de $\mathcal{N}$ :}

Sea $B$ un conjunto acotado de $L^{2}\left(0, T ; H^{-1}(\Omega)\right)$. Si $y \in L^{2}\left(0, T ; H^{-1}(\Omega)\right)$, la solución $u=\mathcal{N}(y) \mathrm{de}$

$$
\begin{cases}u^{\prime}-\Delta u+F(y) u+f(0)=0 & , \text { en } Q \\ u=v & , \text { sobre } \Sigma \\ u(0)=u^{0} & , \text { en } \Omega\end{cases}
$$

pertenece a $L^{2}\left(0, T ; L^{2}(\Omega)\right)$. Entonces, la compacidad de $\mathcal{N}$ es consecuencia de la compacidad de la inmersión

$$
L^{2}\left(0, T ; L^{2}(\Omega)\right) \hookrightarrow L^{2}\left(0, T ; H^{-1}(\Omega)\right)
$$

Acotación del rango de $\mathcal{N}$ :

Dado $y \in L^{2}\left(0, T ; H^{-1}(\Omega)\right)$, el control $v$ del sistema (4.9) es, por el Teorema 3.1, uniformemente acotado en $L^{2}\left(0, T ; L^{2}(\Gamma)\right)$, i.e.,

$$
\|v\|_{L^{2}\left(0, T ; L^{2}(\Gamma)\right)} \leq C
$$


siendo $v_{j}=-\frac{\partial \hat{\varphi}_{j}}{\partial v}$ el control dado por el Teorema 3.1, donde $\hat{\varphi}_{j}$ es solución de

$$
\begin{cases}-\hat{\varphi}_{j}-\Delta \hat{\varphi}_{j}+F\left(y_{j}\right) \hat{\varphi}_{j}=0 & , \text { en } Q \\ \hat{\varphi}_{j}=0 & \text {, sobre } \Sigma \\ \hat{\varphi}_{j}(T)=\hat{\varphi}_{j} & \text {, en } \Omega\end{cases}
$$

con dato inicial $\hat{\varphi}_{j}^{0}$ minimizando el correspondiente funcional $J_{j}$.

Como consecuencia de la Proposición 3.3, $\left(\hat{\varphi}_{j}^{0}\right)_{j}$ es uniformemente acotado en $H_{0}^{1}(\Omega)$. Luego, existen subsucesiones tales que

$$
\hat{\varphi}_{j}^{0} \rightarrow \hat{\varphi}^{0} \text { débilmente en } H_{0}^{1}(\Omega)
$$

Argumentando como en la Proposición 3.3, deducimos que .

$$
v_{j}=-\frac{\partial \hat{\varphi}_{j}}{\partial \nu} \rightarrow \frac{-\partial \hat{\varphi}}{\partial v}=v \text { fuertemente en } L^{2}\left(0, T ; L^{2}(\Gamma)\right)
$$

donde $\hat{\varphi}$ es solución de

$$
\begin{cases}-\hat{\varphi}^{\prime}-\Delta \hat{\varphi}+F(y) \hat{\varphi}=0 & \text {, en } Q \\ \hat{\varphi}=0 & \text {, sobre } \Sigma \\ \hat{\varphi}(T)=\hat{\varphi}^{0} & , \text { en } \Omega\end{cases}
$$

Usando la dependencia continua de $u$ en relación a las condiciones iniciales y de frontera, se muestra que

$$
u_{j} \rightarrow u \text { en } L^{2}\left(0, T ; H^{-1}(\Omega)\right)
$$

siendo $u$ la solución del sistema

$$
\begin{cases}u^{\prime}-\Delta u+F(y) u+f(0)=0 & , \text { en } Q \\ u=v & , \text { sobre } \Sigma \\ u(0)=u^{0} & , \text { en } \Omega\end{cases}
$$

y además tenemos,

$$
\left\|u(T)-u^{1}\right\|_{H^{-1}(\Omega)}<\varepsilon .
$$


Estimativas clásicas de energía para el sistema (4.9) permiten demostrar que

$$
\|u(y)\|_{L^{2}\left(0, T ; L^{2}(\Omega)\right)} \leq C
$$

desde que el potencial considerado es uniformemente acotado.

Esto concluye la demostración del Teorema 4.1.

\section{COMENTARIOS FINALES}

\subsection{Principio de Continuación Única}

El Principio de Continuación Única que hemos utilizado establece que si $\varphi$ es solución del sistema

$$
\begin{cases}-\varphi^{\prime}-\Delta \varphi+a \varphi=0 & , \text { en } Q \\ \varphi=0 & , \text { sobre } \Sigma \\ \varphi(T)=\varphi^{0} & , \text { en } \Omega\end{cases}
$$

y

$$
\frac{\partial \hat{\varphi}}{\partial \nu}=0 \text { en casi todo punto de } \Sigma=\Gamma \times(0, T)
$$

entonces

$$
\varphi=0 \text { en casi todo punto de } Q=\Omega \times(0, T)
$$

\subsection{Control frontera localizado}

En el problema que hemos presentado, el control $v$ actúa sobre la totalidad de la frontera $\Gamma$, sin embargo, podríamos considerar el problema de control

$$
\begin{cases}u^{\prime}-\Delta u+f(u)=0 & , \text { en } Q \\ u=v \mathbf{1}_{\Gamma_{0}} & , \text { sobre } \Sigma \\ u(0)=u^{0} & , \text { en } \Omega\end{cases}
$$

donde $\mathbf{1}_{\Gamma_{0}}$ denota la función característica del abierto $\Gamma_{0} \subset \Gamma$. Es decir, el control $v$ está localizado sobre una parte abierta $\Gamma_{0}$ de la frontera $\Gamma$.

Todo lo anteriormente expuesto puede adaptarse a este problema de control frontera localizado sin mayores cambios. Desde el punto de vista de las aplicaciones, este problema resulta ser más interesante, pues demuestra que es posible conducir la solución $u$ arbitrariamente cerca del estado $u^{1}$ mediante un control que actúa únicamente sobre una parte de la frontera. No hay ninguna restricción en relación a la geometría de $\Gamma_{0}$.

Un cambio natural para adaptar lo aquí expuesto a este problema de control localizado es en la definición del funcional $J$, el mismo que debería definirse como

$$
J\left(\varphi^{0}\right)=\frac{1}{2} \int_{0}^{T} \int_{\Gamma_{0}}\left(\frac{\partial \varphi}{\partial v}\right)^{2} d \Gamma d t+\varepsilon\left\|\varphi^{0}\right\|_{H_{0}^{1}(\Omega)}-\left\langle u^{1}, \varphi^{0}\right\rangle_{H^{-1}(\Omega) \times H_{0}^{1}(\Omega)}
$$




\section{REFERENCIAS BIBLIOGRÁFICAS}

[1] CABANILlAS Z., V. R., MENEZES, S. B., Null Controllability for the Semilinear Heat Equation in Unbounded Domains. PESQUIMAT, Vol. 4, Nº 2, pp. 35-54. (2001).

[2] FABRE, C., PUEL, J. P., ZUAZUA, E., Approximate controllability of the semilinear heat equation. Proc. Royal Soc. Edinburgh, 125A, pp. 31-61. (1995).

[3] FABRE, C., Uniqueness results for Stokes equations and their consequences in linear and non-linear control problems. ESAIM: COCV, 1, pp. 267-302. (1996).

[4] FERNÁNDEZ, L. A., ZUAZUA, E., Approximate controllability for the semilinear heat equation involving gradient terms. J. Optim. Theory Appls. Vol. 1, No 2, pp. 307-328. (1999).

[5] LIONS, J. L., Remarques sur la contrôlabilité approchée. Jornadas Hispano-Francesas de Control de Sistemas Distribuidos, Universidad de Málaga, España, pp. 77-87. (1991).

[6] LIONS, J. L., MAGENES, E., Problémes aux Limites non Homogénes. Vol. 2. Dunod, Paris. (1968).

[7] ZUAZUA, E., Exact boundary controllability for the semilinear wave equation, Nonlinear Partial Differential Equation and their Applications. Vol. X, H. Brezis y J. L. Lions eds., Pitman, pp. 357-391. (1991).

[8] ZUAZUA, E., Finite dimensional null controllability of the semilinear heat equation. J. Math. Pures et Appl., 76, pp. 570-594. (1997).

[9] ZUAZUA, E., Approximate Contrallability for semilinear heat equation with globally Lipschitz nonlinearities, preprint. Universidad Complutense. Madrid España. 